\title{
High Carbohydrate Content in Snap Bean Leaves Stimulates Chinese Rose Beetle Feeding
}

\author{
Sheldon C. Furutani and Lorna H. Arita \\ College of Agriculture, University of Hawaii at Hilo, Hilo, HI 96720-4091 \\ Mike A. Nagao \\ Hawaii Agricultural Experiment Station, University of Hawaii, 461 West \\ Lanikaula Street, Hilo, HI 96720
}

Additional index words. Phaseolus vulgaris, Adoretus sinicus, feeding stimulant

\begin{abstract}
Chinese rose beetle (CRB) (Adoretus sinicus Burmeister) preferential feeding based on leaf carbohydrate content was determined using 'Hawaiian Wonder' snap bean (Phaseohs vulgaris $\mathbf{L}$.) plants exposed to $\mathbf{0 \%}, \mathbf{4 0 \%}$, or $\mathbf{8 0 \%}$ shade for $\mathbf{2}$ days. Plants exposed to $0 \%$ shade before CRBs fed had $8.5 \%$ leaf area consumed compared with $2.8 \%$ and $3.6 \%$ for $40 \%$ and $80 \%$ shade, respectively. Leaves exposed to $0 \%$ shade had a higher carbohydrate content than leaves exposed to $40 \%$ and $80 \%$ shade. Darkening the apical and subtending leaf with aluminum foil for 1 day before CRBs fed shifted CRB feeding from heavy feeding on the apical leaf and light feeding on the subtending leaves to a reversed feeding pattern-light feeding on the apical leaf and heavy feeding on the subtending leaves. Three snap bean cultivars grown under similar environmental conditions were compared to 'Hawaiian Wonder' using a unifoliate split-leaf technique. Cultivars with a high endogenous carbohydrate content in their leaves had a higher percentage of leaf area consumed by CRBs than cultivars with low endogenous carbohy drates. This report suggests that high endogenous carbohydrate content in leaves stimulates CRB feeding.
\end{abstract}

In Hawaii, the Chinese rose beetle (CRB) is a common pest of $>255$ plant species, including many agriculturally important crops (Habeck, 1963). CRBs feed during early evening hours, and their damage is characterized by distinct interveinal defoliation of host plant leaves-damage that can ultimately affect plant maturation and yield (Furutani et al., 1990).

Since CRBs have an extensive host range, Arita et al. (1988) suggested that the stimulus used to initiate and sustain beetle feeding must be a fairly common product found in host plant leaves. Furutani and Arita (1990) suggested that CRBs feed on carbohydrates. In similar in vitro studies, Ladd (1986) reported that several common carbohydrates, including sucrose, maltose, fructose, and glucose, were strong phagostimulants for the adult Japanese rose beetle (Popillia japonica Newman).

Previous CRB feeding studies demonstrated that CRBs preferred plant material

Received for publication 24 Apr. 1992. Accepted for publication 23 Oct. 1992. This research was supported, in part, by a grant from the Agricultural Development in the Pacific Project. We are grateful to Mach and Ray Fukada, Dee Okuhara, and Jill Yokoyama for their technical assistance. The use of trade names in this publication does not imply endorsement by the Univ. of Hawaii College of Agriculture of the products named, nor criticism of similar ones not mentioned. The cost of publishing this paper was defrayed in part by the payment of page charges. Under postal regulations, this paper therefore must be hereby marked advertisement solely to indicate this fact. treated with foliar applications of (2chloroethyl) phosphonic acid (ethephon) (Arita et al., 1988; Furutani and Nagao, 1986). Furutani and Arita ( 1990) proposed that, while leaf carbohydrates may be a general phagostimulant, carbohydrate quantity is of major importance in the beetles' selection of a food source. Similarly, McKibben et al. (1985) and Parrott et al. (1989) found that there was a relationship between the quantity of an ester extract in host plants and the feeding response of the cotton boll weevil (Anthonomus grandis Boheman).

Thus, the objectives of this study were to observe the effect of shade, leaf position, and leaf carbohydrate content of snap beans on CRB feeding.

Shade treatment. 'Hawaiian Wonder' snap bean (Seed Program, Dept. of Horticulture, Univ. of Hawaii, Honolulu) plants were started from seed in a fiberglass greenhouse (Furutani and Arita, 1990). When plants had three ranks of trifoliate leaves ( $\approx 25$ days after sowing), five plants (one per pot) were placed in a screened cage $(1.0 \mathrm{~m}$ high $\times 0.7 \mathrm{~m}$ wide $\mathrm{x} 2.0$ $\mathrm{m}$ long) under full sun or in a shadehouse covered with $40 \%$ or $80 \%$ polypropylene shadecloth $\left(1500,810\right.$, and $214 \mu \mathrm{mol} \cdot \mathrm{m}^{-2} \cdot \mathrm{s}^{-1}$, respectively). Light readings were taken at 1200 HR on a cloudless day. The mean air temperature in the cages was $25 \pm 7 \mathrm{C}$.

Two days afterplants were placed in cages, 25 nonsexed CRBs (collected from the field the previous evening) were placed into each cage between 1600 and $1800 \mathrm{HR}$ and left for 24 h. Plants were removed from the cages and the percent leaf area consumed was quantified with a leaf area meter (LI-3000A; LI-COR, Lincoln, Neb.). The experiment was designed as a randomized complete block with three replicate cages per shade treatment. The experiment was performed seven times; the data from all experiments were pooled. Experiments were conducted between Oct. 1991 and Feb. 1992 at the College of Agriculture Farm Laboratory, Panaewa, Hawaii. Daylight was between 0600 to $1800+0100 \mathrm{HR}$. The data were analyzed by polynomial regression to investigate the trend in percent leaf area consumed by CRBs after shading the plants.

Leaf carbohydrate content was determined for plants grown under each shade treatment as described below. The total carbohydrate content measurement for each treatment was based on 20 replicate plants. The experiment was arranged in a randomized complete block and analyzed by linear regression to investigate the trend in leaf endogenous carbohydrate content resulting from shading.

Foil treatment. Snap bean plants prepared as described above were grown in a fiberglass greenhouse-under $0 \%$ shade. Plants weregrown until four ranks of leaves (order of naming leaves acropetally : unifoliate and trifoliates 1 , 2 , and 3) were visible. Trifoliates 2 and 3 were kept dark by covering the leaves with a single wrapping of aluminum foil between 0800 and 0900 HR. The unifoliate and first trifoliate leaves were not wrapped in foil. The foil wrappings were removed that evening between 1600 and 1800 HR. CRB preferential feeding was determined by placing a single plant in a woodenframed cage $(38 \times 38 \times 62 \mathrm{~cm})$ screened with organdy cloth. Ten nonsexed CRBs (collected from the field the previous evening) were placed in the cage between 1600 and $1800 \mathrm{HR}$, and the cage was then placed in a darkened chamber at $25 \mathrm{C}$. Nonwrapped snap bean plants were used as controls. After $24 \mathrm{~h}$, the unifoliate and trifoliate leaves were removed, and the leaf area consumed by CRBs was determined as described above. Each treatment consisted of 30 replicate plants. Leaf carbohydrate content was determined as described below. The experiment was completely randomized; treatment means and SE values were calculated for percent leaf area consumed and leaf endogenous carbohydrate content after wrapping leaves.

Cultivar trials. 'Kentucky Wonder', 'Green Crop', and 'Blue Lake Bush 274' (W. Atlee Burpee Co., Warminster, Pa.) bean plants were planted in the greenhouse as described under shade treatment and grown unshaded until their unifoliate leaves were fully expanded $(\approx 12$ days). The unifoliates from each cultivar were excised and sectioned along the midrib with a razor blade. A sectioned unifoliate leaf from each cultivar was placed beside a 'Hawaiian Wonder' section (split-leaf arrangement). The midribs of the two unifoliate leaf sections were matched to simulate a whole leaf. The paired sections were placed in a 9-cm petri dish fitted with a single layer of Whatman no. 2 filter paper moistened with $1.0 \mathrm{ml}$ distilled water. One CRB was placed in the petri dish and the dish was placed in a dark chamber at $24 \mathrm{C}$ for $12 \mathrm{~h}$. The unifoliate leaf sections 
were removed from the dish, and the leaf area consumed by the CRB was quantified with a leaf area meter as already described. Each treatment comparison was replicated 30 times. Ten plants from each cultivar were sampled for the total carbohydrate content measurement. Unifoliate split leaves were prepared and CRBs introduced between 1600 and 1800 HR. The experiment was designed as a randomized complete block; data were analyzed by paired two-group $t$ tests to compare percent leaf area consumed and leaf endogenous carbohydrate content between snap bean cultivars.

Total carbohydrate content measurement. Leaf total carbohydrate content was determined by sampling trifoliate and unifoliate leaves. Leaves were analyzed by subsampling all unifoliate and trifoliate leaflets midway from the base to the leaf apex and $5 \mathrm{~mm}$ to the left of the midrib with a 3-mm-diameter cork borer. Total carbohydrate content of leaves (expressed as micrograms of sucrose equivalent $/ 5 \mathrm{~mm}^{2}$ leaf area) was determined with Dreywood's anthrone reagent as described by Morris (1948).

Influence of shade treatment on feeding. Snap bean plants exposed to $0 \%$ shade before CRBs fed had $\approx 8.5 \%$ of the leaf area consumed compared to $2.8 \%$ to $3.6 \%$ for $40 \%$ and $80 \%$ shade, respectively. The relationship between percent leaf area consumed and percent shade was quadratic $\left(R^{2}=0.23, P=0.01\right)$ (Fig. 1). Plants grown in $0 \%$ shade also had the highest leaf carbohydrate content, expressed as sucrose equivalents (Fig. 2). Trend analysis suggests that there is a linear relationship between leaf carbohydrate content and percent shade ( $R^{2}=0.81, P=0.01$ ).

Effect of foil treatment on feeding. The unifoliates and trifoliates 1 and 2 had only $1.9 \%$ to $2.8 \%$ feeding damage, while trifoliate 3 had $6.2 \%$ feeding damage (Fig. 3). Nonwrapped leaf carbohydrate content for trifoliates 1,2 , and 3 was from 59.2 to $61.2 \mu \mathrm{g}$ sucrose eq $/ 5 \mathrm{~mm}^{2}$ leaf area, while the unifoliates had the lowest carbohydrate content-47.1 $\mu \mathrm{g}$ sucrose eq $/ 5 \mathrm{~mm}^{2}$ leaf area (Fig. 4). Wrapping trifoliates 2 and 3 in foil significantly decreased leaf feeding at trifoliate 3 compared to feeding at the nonwrapped leaves. The unifoliates and trifoliate 1 had a higher percent leaf area consumed by CRBs when trifoliates 2 and 3 were wrapped. Leaf carbohydrate content for wrapped and nonwrapped snap bean plants was similar, except for trifoliates 2 and 3 , which had a $>50 \%$ decrease in carbohydrate content compared to the nonwrapped control (Fig. 4). The total percent leaf area consumed of wrapped $(13.5 \pm 0.73 \mathrm{SE})$ compared to nonwrapped $(13.0 \pm 0.82 \mathrm{SE})$ plants was not significant.

Effect of cultivar on feeding. 'Blue Lake Bush 274' and 'Kentucky Wonder' snap bean cultivars had higher percent leaf area consumed than 'Hawaiian Wonder' (Table 1). Both cultivars also had higher leaf carbohydrate contents than 'Hawaiian Wonder'. There was no difference between leaf carbohydrate content or percent leaf area consumed for 'Green Crop' and 'Hawaiian Wonder'.

Wrapping trifoliates 2 and 3 in foil during

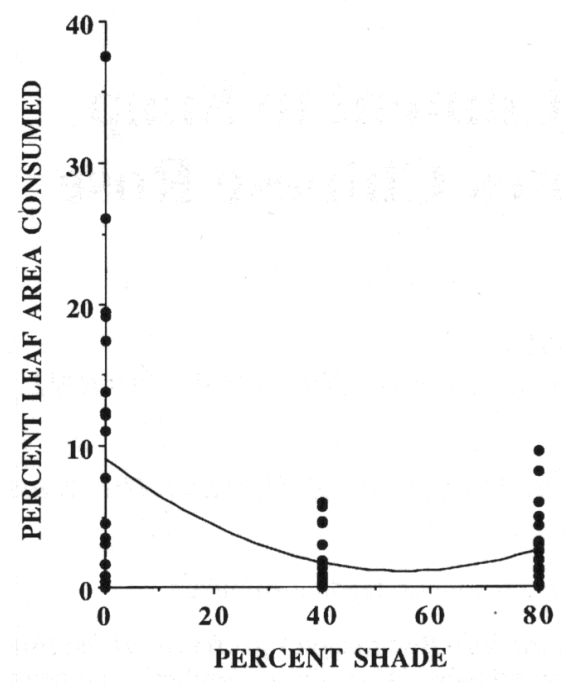

Fig. 1. Relationship between exposing snap bean plants to $0 \%, 40 \%$, or $80 \%$ shade (S) for 2 days and percent leaf area consumed by the Chinese rose beetle. Regression equation: percent leaf area consumed $=9.1-0.3(\mathrm{~S} \%)+0.0026(\mathrm{~S} \%)^{2}$ $\left(R^{2}=0.23, P=0.01\right)$.

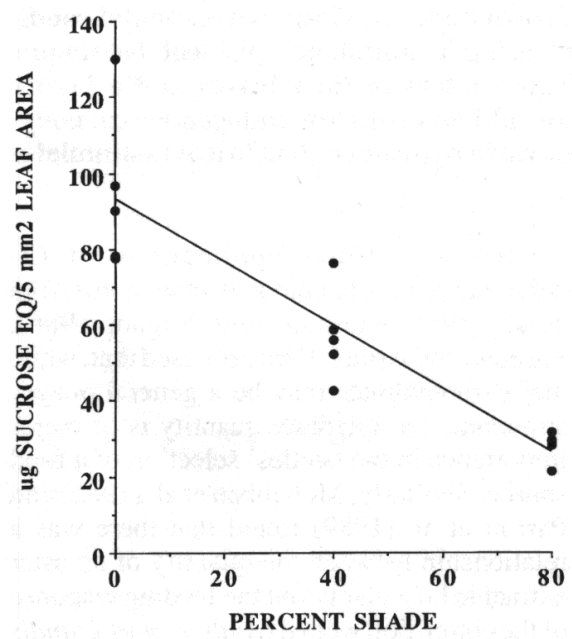

Fig. 2. Relationship between exposing plants to $0 \%$, $40 \%$, or $80 \%$ shade (S) for 2 days and accumulated endogenous carbohydrate content of snap bean leaves at 1600 to 1800 HR. Regression equation: accumulated endogenous carbohydrate content $=93.2-0.8(\mathrm{~S} \%)\left(R^{2}=0.81\right.$, $P=0.01)$.

daylight hours kept the leaves from photosynthesizing. Consequently, during evening hours, when CRBs fed, these leaves were abnormally low in endogenous carbohydrates. In response to the lowered carbohydrates, CRBs shifted their feeding to the lower leaves where the carbohydrate content was higher. Apparently, CRBs are able to assess leaf endogenous carbohydrate content. CRBs may determine carbohydrate content by sampling the leaves, since tiny feeding holes were observed on the wrapped leaves.

Differences in the carbohydrate content of leaves were observed in snap bean cultivars grown under the same environmental conditions. A higher carbohydrate content in the

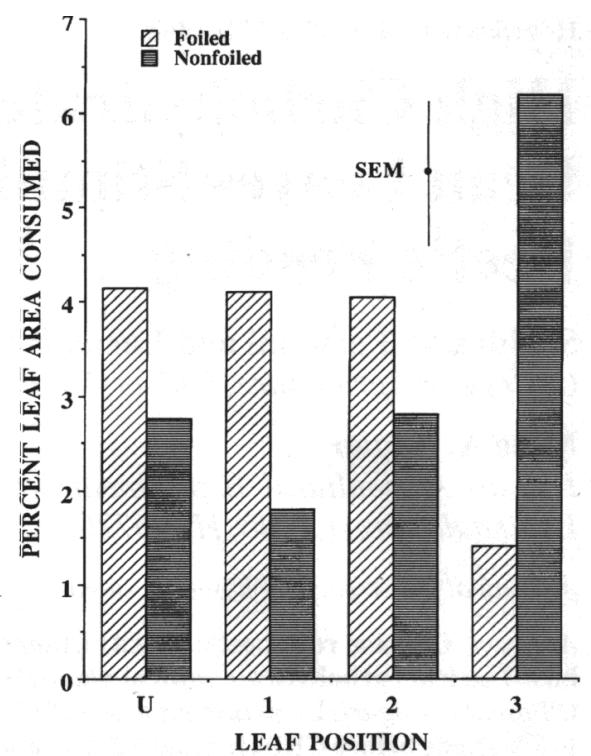

Fig. 3. Comparison of percent leaf area consumed in relation to leaf position [order of leaves acropetally: unifoliates (U), trifoliates 1,2 , and $3]$ for nonwrapped and wrapped (trifoliates 2 and 3 were wrapped; trifoliate 1 and unifoliates were not wrapped) snap bean plants. Trifoliates were wrapped (kept dark) for 1 day; foil was removed just before Chinese rose beetle feeding.

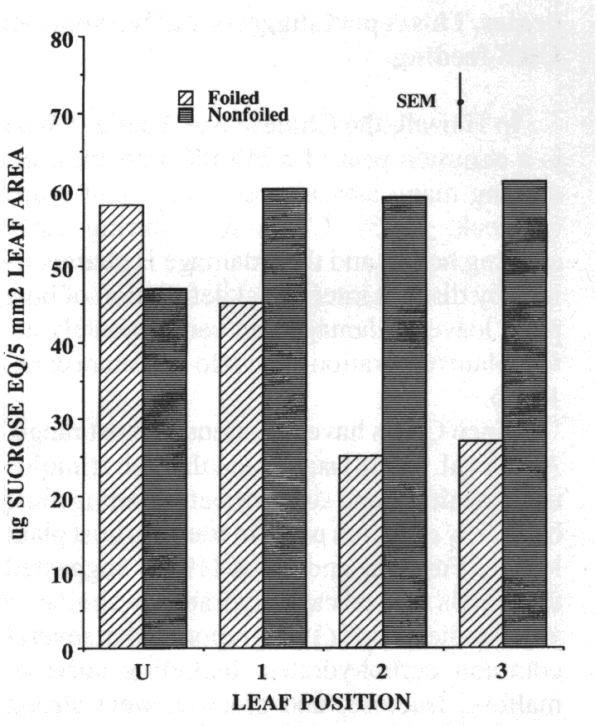

Fig. 4. Comparison of endogenous carbohydrate content in relation to leaf position [order of leaves acropetally: unifoliate (U), trifoliates 1 , 2, and 3] for nonwrapped and wrapped (trifoliates 2 and 3 were wrapped; trifoliate 1 and unifoliates were not wrapped) snap bean plants. Trifoliates were wrapped (kept dark) for 1 day; foil was removed just before carbohydrate analysis.

leaves resulted in a higher percent leaf area consumed by CRBs. Clearly, CRBs select for and feed on those cultivars possessing a high level of leaf carbohydrates. We presume that the CRBs are selecting a mobile carbohydrate source, such as starch grains accumulated via photosynthesis. We also presume that the carbohydrate source is not structural cellulose, because Furutani and Arita (1990) reported 
Table 1. Leaf carbohydrate content and percent leaf area consumed by the Chinese rose beetle in split-leaf analyses of three snap bean cultivars compared with 'Hawaiian Wonder'.

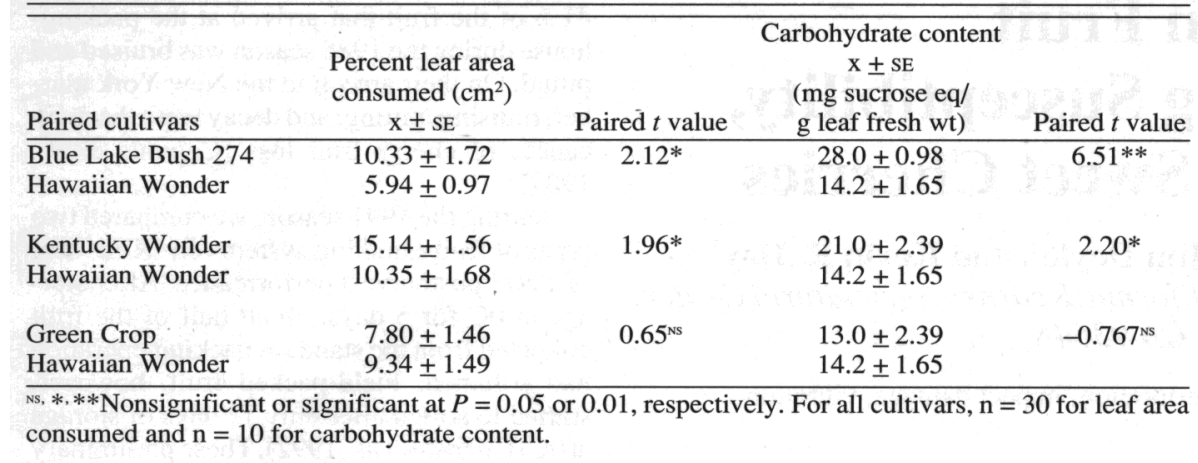

higher percent leaf area consumed on snap bean leaf explants treated with exogenous carbohydrates.

Determining plant constituents that cause insects such as CRBs to select and feed on a particular plant can facilitate pest management. Cultural practices, breeding plant selections that can lower the endogenous carbohy-

drate content in their leaves during early evening hours when CRBs feed, or both may be nonchemical alternatives for controlling the detrimental feeding effects of this widespread tropical insect pest of leaves.

\section{Literature Cited}

Arita, L.H., SC. Furutani, and J.J. Moniz. 1988 Preferential feeding by the Chinese rose beetle (Coleoptera: Scarabaeidae) on ethephon treated plants. J. Econ. Entomol. 81:1373-1376.

Furutani, SC. and L.H. Arita. 1990. Effect of light exposure and carbohydrate content of snap bean leaves on Chinese rose beetle (Coleoptera:
Scarabaeidae) feeding. J. Econ. Entomol. 83:2022-2025

Furutani, S.C., L.H. Arita, and J.K. Fujii. 1990. Relationship between simulated Chinese rose beetle (Coleoptera: Scarabaeidae) feeding and photosynthetic rate reduction. Proc. Hawaii Entomol. Soc. 30:97-104.

Furutani, S.C. and M.A. Nagao. 1986. Influence of daminozide, gibberellic acid, and ethephon on flowering, shoot growth, and yield of ginger. HortScience 23:428-429.

Habeck, D.H. 1963. Descriptions of immature stages of the Chinese rose beetle, Adoretus sinicus Burmeister (Coleoptera: Scarabaeidae). Proc. Hawaii Entomol. Soc. 18:251-258.

Ladd, T.L. 1986. Influence of sugars on the feeding response of Japanese beetle (Coleoptera: Scarabaeidae). J. Econ. Entomol. 79:668-671.

McKibben, G.H., M.J. Thompson, W.L. Parrot, A.C. Thompson, and W.R. Lusby. 1985. Identification of feeding stimulants for boll weevils from cotton buds and anthers. J. Chem. Ecol. 11:1229-1238.

Morris, D.L. 1948. Quantitative determination of carbohydrates with Dreywood's anthrone reagent. Science 107:254-255.

Parrott, W.L., G.H. McKibben, J.B. Robbins, and E.J. Villavaso. 1989. Feeding response of the boll weevil (Coleoptera: Curculionidae) to ester extracts of host plants. J. Econ. Entomol. 82:449453. 